\title{
DISTRIBUIÇÃO SAZONAL DE LARVAS INFECTANTES DE CIATOSTOMÍNEOS (Nematoda-Cyathostominae) NA BAIXADA FLUMINENSE DO RIO DE JANEIRO
}

\author{
Simone Quinelato, ${ }^{1}$ Melissa Carvalho Machado do Couto, ${ }^{1}$ Flávio Couto Cordeiro, ${ }^{2}$ Ivan Barbosa \\ Machado Sampaio, ${ }^{3}$ e Maria de Lurdes de Azevedo Rodrigues ${ }^{4}$ \\ 1. Mestranda bolsista Capes, Curso de Pós-Graduação em Ciências Veterinárias (CPGCV), URRRJ. E-mail: squinelato@ufrrj.br; melcouto@ufrrj.br \\ 2. Doutorando bolsista, Capes, Programa de Pós-Graduação em Química Orgânica, UFRRJ. E-mail: fcordeiro@ufrrj.br \\ 3. Professor titular, Departamento de Zootecnia, Escola de Medicina Veterinária, UFMG. E-mail: ivan@vet.ufmg.br \\ 4. Departamento de Parasitologia Animal, Instituto de Veterinária, CPGCV. E-mail: lurdesar@ufrrj.br
}

\section{RESUMO}

Sob condições de clima tropical da Baixada Fluminense do Rio de janeiro, foi desenvolvido um experimento com amostras de fezes depositadas em gramínea Tyfton 85 (Cynodon spp. Cv.Tifton 85), no início de cada estação do ano. O objetivo do estudo foi avaliar a distribuição das larvas infectantes $\left(\mathrm{L}_{3}\right)$ de ciatostomíneos nas fezes, na gramínea e no solo, verificando o horário de maior recuperação de larvas. As coletas iniciaram-se uma semana após cada depósito e a partir daí de quinze em quinze dias durante as estações. Maior número de larvas foi encontrado na gramínea no outono e no inverno, meses com predominância na estação seca, e menor número de larvas foi encontrado na primavera e principalmente no verão, meses com predominância na estação chuvosa. No horário das 8 horas, foi recuperado o maior número de larvas. Não se observou diferença significativa entre os horários de coleta para cada estação do ano. Reduzido número de larvas foi recuperado nos $10 \mathrm{~cm}$ do solo analisado. As condições climáticas da região estudada permitiram o desenvolvimento e a sobrevivência de $\mathrm{L}_{3}$ de ciatostomíneos na massa fecal, assim como a migração para a gramínea, durante todo o ano, com maior risco de infecção para os equinos no outono e no inverno.

PALAVRAS-CHAVES: Ciatostomíneos, clima tropical, ecologia, Tifton 85.

\section{ABSTRACT}

\section{SEAZONAL DISTRIBUTION OF CYATHOSTOMIN (Nematoda-Cyathostominae) INFECTIVE LARVAE IN BAIXADA FLU-} MINENSE, RIO DE JANEIRO, BRAZIL

Distribution of cyathostomin infective larvae $\left(\mathrm{L}_{3}\right)$ in feces, soil and in Tifton 85 (Cynodon spp. cv. Tifton 85) pasture was studied in the different seasons under tropical climate of Baixada Fluminense, Rio de Janeiro state, Brazil. The aim of this study was to evaluate the distribution of $\mathrm{L}_{3}$ in the feces, pasture and soil, verifying the time of higher larvae recovery. The collections began one week after deposit, and then every 15 days. $\mathrm{L}_{3}$ were recovered in higher number in the fall and winter, dry period, when compared to spring and mainly the summer, rainy period. More larvae were recovered at 8:00 o'clock, although no statistic difference was observed among the collection times for each season. The soil did not demonstrate to be a potential $\mathrm{L}_{3}$ reservoir, considering the low larvae recovery. The environmental conditions of the studied area rendered possible the $\mathrm{L}_{3}$ development and survival in fecal mass, as well as the migration for the pasture, making possible the infection of horses.

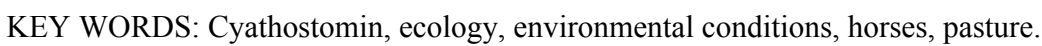




\section{INTRODUÇÃO}

O uso intensivo de drogas anti-helmínticas tem causado dramáticas mudanças na prevalência e biologia dos nematoides de equinos. Desde as últimas décadas, vêm sendo recomendadas medidas como redução na intensidade dos tratamentos, tratamentos seletivos e aumento na vigilância parasitária (DUNCAN \& LOVE, 1991; GOMEZ \& GEORGI, 1991; KRECEK et al., 1994; KAPLAN, 2002; MATTHEE \& MCGEOCH, 2004), a fim de se reduzir o desenvolvimento de resistência às drogas.

Entre os fatores que influenciam a resistência dos nematoides aos anti-helmínticos, o mais importante é o tamanho do refugium parasitário (VAN WYK, 2001; KAPLAN, 2004), constituído principalmente pelos estágios de vida livre presentes na pastagem (NIELSEN et al., 2007).

A dinâmica populacional dos helmintos gastrintestinais e a interferência dos efeitos relacionados ao ambiente, tais como a estação do ano, temperatura e chuva, têm sido um importante objeto de estudo. Alguns trabalhos vêm sendo realizados em regiões de clima tropical (MFITILODZE \& HUTCHINSON, 1988; HUTCHINSON, et al., 1989; QUINELATO, et al., 2007; RODRIGUES et al., 2008; COUTO et al., 2008; COUTO et al., 2009) e subtropical (ENGLISH, 1979a; ENGLISH, 1979b; CRAIG et al., 1983; COURTNEY \& ASQUITH, 1985; BAUDENA et al., 2000a; BAUDENA et al., 2000b). No entanto, ainda não está totalmente esclarecida a dinâmica populacional destes no ambiente (BUCKNELL etal., 1995; BAUDENA et al., 2000a; LANGROVÁ et al., 2003). Dessa forma, para o desenvolvimento de eficientes programas de controle, informações sobre a distribuição dos estágios pré-parasíticos são essenciais, visto que possibilitam a avaliação do risco de infecção para os animais.

O objetivo deste estudo foi avaliar a distribuição das larvas infectantes de ciatostomíneos nas fezes, gramínea e solo nas diferentes estações do ano e o horário de maior recuperação de larvas em condições de clima tropical da Baixada Fluminense do Rio de Janeiro.

\section{MATERIAL E MÉTODOS}

\section{Localização}

Para a realização do estudo foi montado um canteiro experimental com a gramínea Tifton $85(C y$ - nodon spp. cv. Tifton 85), de dimensões 11,5 x 1,0 m, localizado na Estação para Pesquisas Parasitológicas W. O. Neitz, do Departamento de Parasitologia Animal do Instituto de Veterinária da Universidade Federal Rural do Rio de Janeiro (UFRRJ). A área está localizada a $22^{\circ} 41^{\prime}$ de latitude Sul e $43^{\circ} 41^{\prime}$ de longitude Oeste, à altitude de $33 \mathrm{~m}$. O clima é do tipo Aw (tropical úmido), de acordo com a classificação de Köppen. O solo da região estudada é classificado como Planossolo Háplico (EMBRAPA, 2006).

\section{Experimento}

O estudo foi desenvolvido no período de setembro/2006 a setembro/2007. Amostras de fezes foram obtidas de dois equinos naturalmente infectados mantidos na Estação para Pesquisas Parasitológicas W. A. Neitz. No início de cada estação do ano, coletou-se $1 \mathrm{~kg}$ de fezes de cada animal doador. Posteriormente, o material coletado foi dividido em duas amostras de $500 \mathrm{~g}$ por animal, totalizando quatro repetições, que foram depositadas no canteiro experimental. Uma semana após cada depósito, coletaram-se amostras de fezes, gramínea e solo, rotina que posteriormente ocorreu a cada quinze dias até o final de cada estação. Procedeu-se às coletas de fezes $( \pm 2 \mathrm{~g})$, gramínea $(0-20 \mathrm{~cm}$ de altura) e solo $(10 \mathrm{~cm})$ em dois horários diferentes ( 8 e17 horas) e em três pontos distintos ao redor da massa fecal. $\mathrm{O}$ processamento das amostras seguiu a metodologia de QUINELATO et al. (2007).

Os dados climáticos foram fornecidos pelo posto Agrometeorológico da Estação Ecológica Agrícola de Seropédica - INMET/PESAGRO - RJ e a temperatura do solo foi mensurada a cada coleta do canteiro experimental.

As estações do ano foram definidas por primavera (23/09-21/12), verão (22/12-19/03), outono (20/03-20/06) e inverno (21/06-22/09). O período de chuvas é, normalmente, compreendido entre outubro e março e o período seco, entre abril e setembro.

Análise estatística

Para a análise de variação do número médio de $\mathrm{L}_{3}$ (larvas infectantes) recuperadas das fezes, da gramínea e do solo nos diferentes horários de coleta, em cada estação do ano, foi utilizado o teste de Kruskal-Wallis $(\mathrm{p}<0,05)(Z A R, 1999$; SAMPAIO, 
2007), pelo programa BioEstat (AYRES et al., 2005), assim como para a análise dos valores médios de OPG (número de ovos por grama de fezes) em cada estação do ano. Analisou-se a correlação entre o número médio de larvas recuperadas das fezes, da gramínea e do solo e as variáveis ambientais temperatura média do ar, temperatura média do solo e índice pluviométrico total pelo teste de coeficiente de correlação de Spearman $(p<0,05)$ (ZAR, 1999; SAMPAIO, 2007), BioEstat (AYRES et al., 2005).

\section{RESULTADOS}

Distribuição das $\mathrm{L}_{3}$ nas fezes

O OPG dos animais doadores variou durante o experimento (Tabela 1) e não se observou diferença estatística entre as estações do ano. A dinâmica de recuperação de larvas infectantes nas diferentes estações do ano, em cada horário de coleta, pode ser observada na Figura 1. O número médio de larvas recuperadas no outono $\left(593.469 \mathrm{~L}_{3} \cdot \mathrm{kg}^{-1} \cdot \mathrm{ms}\right)$ e inverno (311.045 $\left.\mathrm{L}_{3} \cdot \mathrm{kg}^{-1} . \mathrm{ms}\right)$ foi mais elevado que na primavera $\left(24.049 \mathrm{~L}_{3} \cdot \mathrm{kg}^{-1} \cdot \mathrm{ms}\right)$ e no verão $\left(1.094 \mathrm{~L}_{3} \cdot \mathrm{kg}^{-1}\right.$. $\mathrm{ms})(\mathrm{p}>0,05)$. Em relação aos horários de coleta, para cada estação do ano, não foi observada diferença significativa. No horário de 8 horas, recuperou-se maior número de $\mathrm{L}_{3}$ nas estações outono e inverno em relação ao verão. Nas coletas realizadas às 17 horas, também se recuperou maior número de larvas no outono e no inverno e menor número na primavera e verão $(p<0,05)$.

Distribuição das $\mathrm{L}_{3}$ na gramínea

Na gramínea Tifton 85, a recuperação média das $\mathrm{L}_{3}$ foi superior no outono $\left(2.550 \mathrm{~L}_{3} \cdot \mathrm{kg}^{-1} \cdot \mathrm{ms}\right) \mathrm{e}$ no inverno $\left(3.502 \mathrm{~L}_{3} \cdot \mathrm{kg}^{-1} \cdot \mathrm{ms}\right)$ em comparação aos valores na primavera $\left(634 \mathrm{~L}_{3} \cdot \mathrm{kg}^{-1} \cdot \mathrm{ms}\right)$ e no verão (655 $\left.\mathrm{L}_{3} \cdot \mathrm{kg}^{-1} \cdot \mathrm{ms}\right)$ (Figura 2) $(\mathrm{p}<0,05)$. Semelhante ao observado para recuperação de larvas nas fezes, não houve diferença estatística entre os horários de coleta da gramínea em cada estação do ano. Diferença significativa foi observada para as $\mathrm{L}_{3}$ recuperadas às 8 horas e 17 horas entre as estações do ano, sendo os maiores valores encontrados no outono e no inverno, quando comparados com os da primavera e verão $(p<0,05)$.
TABELA 1. OPG das massas fecais depositadas ao início de cada estação do ano no período de setembro/2006 a setembro/2007. ( $\mathrm{P}=$ primavera, $\mathrm{V}=$ verão, $\mathrm{O}=$ outono, $\mathrm{I}=$ inverno $)$

\begin{tabular}{lcccc}
\hline & $\mathrm{P}$ & $\mathrm{V}$ & $\mathrm{O}$ & $\mathrm{I}$ \\
\hline Cavalo 1 & 1250 & 1000 & 2900 & 1300 \\
Cavalo 2 & 2250 & 1050 & 1700 & 2500 \\
\hline
\end{tabular}

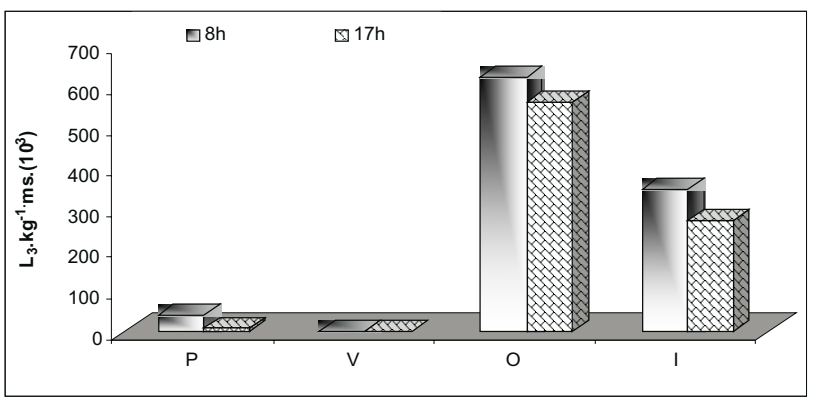

FIGURA 1. Número médio de larvas infectantes recuperadas das amostras de fezes, em cada estação do ano, nos diferentes horários de coleta, no período de setembro/2006 a setembro/2007. ( $\mathrm{P}=$ primavera, $\mathrm{V}=$ verão, $\mathrm{O}=$ outono, $\mathrm{I}=$ inverno)

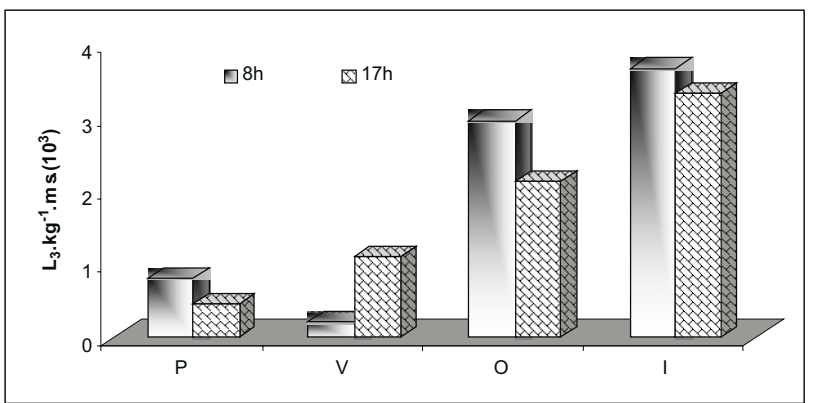

FIGURA 2. Número médio de larvas infectantes recuperadas das amostras de gramínea, em cada estação do ano, nos horários de coleta de 8 e17 horas, no período de setembro/2006 a setembro/2007. $(\mathrm{P}=$ primavera, $\mathrm{V}=$ verão, $\mathrm{O}=$ outono, $\mathrm{I}=$ inverno $)$

Distribuição das $\mathrm{L}_{3}$ no solo

Nas estações outono $\left(308 \mathrm{~L}_{3} \cdot \mathrm{kg}^{-1} \cdot \mathrm{ms}\right)$ e inverno (369 $\mathrm{L}_{3} \cdot \mathrm{kg}^{-1} \cdot \mathrm{ms}$ ) foi observada maior recuperação média de $\mathrm{L}_{3}$ em comparação à primavera $\left(13 \mathrm{~L}_{3} \cdot \mathrm{kg}^{-1}\right.$. $\mathrm{ms}$ ) e verão (19 $\left.\mathrm{L}_{3} \cdot \mathrm{kg}^{-1} \cdot \mathrm{ms}\right)$ (Figura 3 ), porém não se observou diferença significativa entre as estações. Não se verificou diferença estatística nos horários de 8 e17 horas, em cada estação e entre elas. 


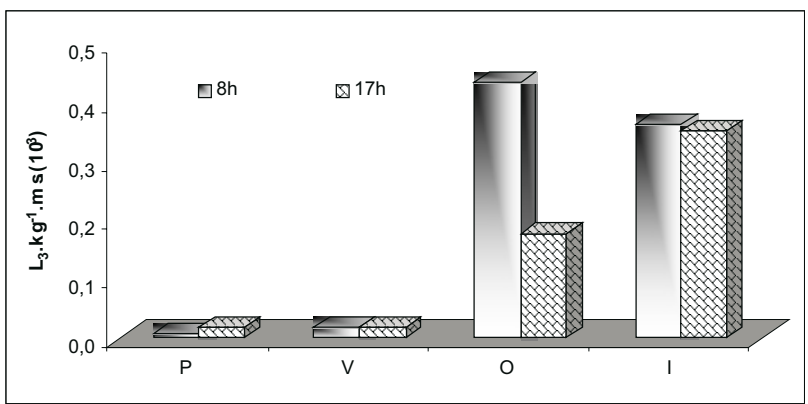

FIGURA 3. Número médio de larvas infectantes recuperadas das amostras de solo, em cada estação do ano, nos diferentes horários de coleta, no período de setembro/2006 a setembro/2007. ( $\mathrm{P}=$ primavera, $\mathrm{V}=$ verão, $\mathrm{O}=$ outono, $\mathrm{I}=$ inverno)

\section{Dados climáticos}

A temperatura do ar e do solo e o índice pluviométrico total para cada estação do ano podem ser observados na Figura 4. O verão foi a estação que apresentou maiores médias de temperatura, tanto do ar quanto do solo, $26,42{ }^{\circ} \mathrm{C}$ e $28,75^{\circ} \mathrm{C}$, respectivamente, e maior índice pluviométrico total $(468,60 \mathrm{~mm})$. As menores médias de temperatura e menor índice pluviométrico total foram observadas no inverno, médias de temperatura do ar de $21,98^{\circ} \mathrm{C}$ e do solo de $22,50{ }^{\circ} \mathrm{C}$ e índice pluviométrico total de $77,40 \mathrm{~mm}$.

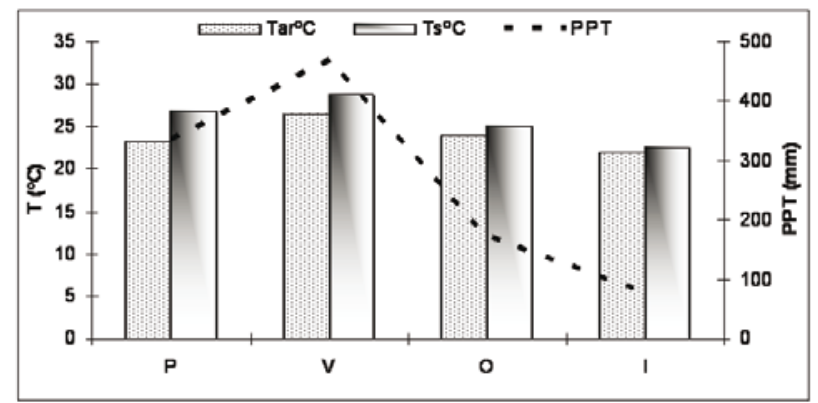

FIGURA 4. Temperatura média do ar, do solo e índice pluviométrico total observado em cada estação do ano durante o período de setembro/2006 a setembro/2007. ( $\mathrm{P}=$ primavera, $\mathrm{V}=$ verão, $\mathrm{O}=$ outono, $\mathrm{I}=$ inverno)

Correlação entre recuperação de larvas e dados climáticos

A análise estatística evidenciou elevada correlação negativa entre o índice pluviométrico e a recuperação de $\mathrm{L}_{3}$ nas fezes, na gramínea e no solo. Fato também observado na correlação entre temperatura do solo e o número de larvas recuperadas. Não houve correlação significativa entre a temperatura do ar e o número de $\mathrm{L}_{3}$ recuperadas, entretanto observou-se maior influência deste parâmetro sobre a recuperação de $\mathrm{L}_{3}$ na gramínea $(|\mathrm{r}|=-0,586)$ (Tabela 2$)$.

TABELA 2. Coeficiente de correlação de Spearman entre o número de larvas recuperadas nas fezes, gramínea e solo com as variáveis climáticas estudadas

\begin{tabular}{cccc}
\hline & PPT $(\mathrm{mm})$ & $\begin{array}{c}\text { Temp. ar } \\
\left({ }^{\circ} \mathrm{C}\right)\end{array}$ & $\begin{array}{c}\text { Temp. solo } \\
\left({ }^{\circ} \mathrm{C}\right)\end{array}$ \\
\hline Fezes & $-0,732^{*}$ & $-0,293^{\mathrm{ns}}$ & $-0,732^{*}$ \\
Gramínea & $-0,878^{*}$ & $-0,586^{\mathrm{ns}}$ & $-0,878^{*}$ \\
Solo & $-0,732^{*}$ & $-0,293^{\mathrm{ns}}$ & $-0,732^{*}$ \\
\hline
\end{tabular}

Teste de coeficiente de correlação de Spearman. $(*=p<0,05)$ (ns $=$ não significativo)

\section{DISCUSSÃO}

As variáveis climáticas da região influenciaram de forma marcante a recuperação das $\mathrm{L}_{3}$ O inverno e o outono foram as estações com maior recuperação de larvas infectantes, com valores mais baixos para temperaturas do ar e do solo, e chuva, em comparação com os valores do verão, estação em que as $\mathrm{L}_{3}$ foram recuperadas em menor quantidade.

Estudos demonstram que o desenvolvimento das larvas infectantes de ciatostomíneos é considerado ótimo em temperaturas entre $10-33^{\circ} \mathrm{C}$ e que a chuva afeta de forma negativa a sobrevivência das $\mathrm{L}_{3}$, embora seja necessária para fornecer a quantidade mínima de umidade para migração das larvas (HUTCHINSON et al., 1989) e para o desenvolvimento das formas pré-infectantes (OGBOURNE, 1972; RUPASHING \& OGBOURNE, 1978; MFILODZE \& HUTCHINSON, 1987).

O bolo fecal atuou como reservatório de larvas, principalmente nas estações mais secas, como no outono e inverno, demonstrando que nessas estações o ressecamento não promove redução intensa do número de larvas, ocorrendo apenas uma diminuição na transmissão das $\mathrm{L}_{3}$ das fezes para a pastagem até que a umidade seja restabelecida novamente, o que salienta a importância não somente do comportamento das $\mathrm{L}_{3}$ na 
pastagem, mas também de sua dinâmica nas fezes. Resultados semelhantes foram relatados anteriormente por OGBOURNE (1972), ENGLISH (1979a), BAUDENA et al. (2000), RAMSEY et al. (2004), KUZMINA et al. (2006), QUINELATO et al. (2007), QUINELATO et al. (2008); COUTO et al. (2008),COUTO et al. (2009) e RODRIGUES et al. (2008). Outro possível reservatório de larvas infectantes é o solo das pastagens. Alguns estudos relatam a capacidade que as $\mathrm{L}_{3}$ de estrongilídeos de equinos têm para migrar ao solo e também a importância de aspectos como tipo de solo e condições climáticas, que podem afetar a viabilidade da larva no solo (HOUSTON et al., 1984). Existem poucas informações e estudos sobre a recuperação de larvas infectantes de estrongilídeos de equinos no solo, sendo essas informações maiores em estudos sobre larvas infectantes de nematoides de bovinos. Mesmo assim, aparecem de forma controversa. Alguns autores afirmam que em condições desfavoráveis as $\mathrm{L}_{3}$ migram a determinadas profundidades do solo, buscando um refúgio (LYAKU et al., 1988). Outros autores discordam, afirmando terem recuperado número reduzido de larvas no solo (ROSE \& SMALL, 1985; CASTRO et al., 2004). Estes resultados são equivalentes aos encontrados neste estudo, em que se recuperaram poucas $\mathrm{L}_{3}$ do solo, em todas estações do ano, em comparação com os valores recuperados nas fezes e na gramínea.

No horário da manhã as larvas foram recuperadas em maior número, demonstrando a importância da umidade e de temperaturas amenas, embora não tenha sido observada diferença estatística entre os horários de coleta em cada estação do ano, concordando com os relatos de LANGROVÁ et al. (2003), que destacaram a importância do orvalho da manhã para a migração das larvas. A comparação do número de larvas recuperadas, em cada horário de coleta, entre as estações do ano, evidenciou menor recuperação de larvas no verão, demonstrando que as condições climáticas da estação são desfavoráveis para a sobrevivência e recuperação das $\mathrm{L}_{3}$.

As fortes chuvas do verão tornaram esta estação crítica para a recuperação das larvas, promovendo a destruição das massas fecais e lixiviação das larvas para longe da pastagem. Já o ressecamento, associado às elevadas temperaturas, principalmente do solo, embora não tenha tido efeito deletério, prejudicou as $\mathrm{L}_{3}$, que não conseguiram escapar das fezes e migrar para a pastagem.
Larvas infectantes de ciatostomíneos foram recuperadas da pastagem durante todas as estações do ano. Demonstra-se, assim, a importância do estudo desse refugium parasitário para o desenvolvimento de programas de controle, levando em consideração a combinação entre fatores climáticos e práticas de pastejo dos animais para a avaliação dos níveis adequados de refugium, evitando tratamentos desnecessários (NIELSEN et al., 2007).

\section{CONCLUSÃO}

As condições ambientais da região estudada possibilitaram o desenvolvimento de $\mathrm{L}_{3}$ de ciatostomíneos na massa fecal, assim como a migração para a pastagem, possibilitando a infecção dos cavalos. Condições de baixo índice pluviométrico, associadas a temperaturas relativamente amenas, podem determinar a sobrevivência de larvas infectantes na massa fecal, representando fonte de contaminação para a pastagem. As $\mathrm{L}_{3}$ foram recuperadas em maior quantidade nas estações de outono e inverno, consideradas secas, e em menor quantidade na primavera e principalmente no verão, estação chuvosa. Maior número de larvas infectantes de ciatostomíneos foi recuperado no horário das 8 horas. $\mathrm{O}$ solo não demonstrou ser potencial reservatório de larvas infectantes de ciatostomíneos..

\section{AGRADECIMENTOS}

Ao Curso de Pós-Graduação em Ciências Veterinárias (CPGCV), à Universidade Federal Rural do Rio do Janeiro (UFRRJ), à Coordenação de Aperfeiçoamento de Pessoal de Ensino Superior (CAPES) e ao Conselho Nacional de Desenvolvimento Tecnológico (CNPq).

\section{REFERÊNCIAS}

AYRES, M.; AYRES JR. M.; AYRES, D. L.; SANTOS, A. S. DOS. BioEstat 4.0: aplicações estatísticas nas áreas das ciências biológicas e médicas. 4. ed. Belém: IOEPA, 2005. 324 p.

BAUDENA, M. A.; CHAPMAN, M. R.; FRENCH, D. D.; KLEI, T. R. Seasonal development and survival of equine cyathostome larvae on pasture in south Louisiana, Veterinary Parasitology, v. 88 , n. 1-2, p. 51-60, 2000a. 
BAUDENA, M. A.; CHAPMAN, M. R.; LARSEN, M.; KLEI, T. R. Efficacy of the namatophagus fungus Duddingtonia flagrans in reducing equine cyathostome larvae on pasture in south Luisiana. Veterinary Parasitology, v. 89, n. 4, p. 219-230, 2000 b.

BUCKNELL, D. G.; GASSER, R. B.; BEVERIDGI, I. The prevalence and epidemiology of gastrointestinal parasites of horses in Victoria, Australia. International Journal of Parasitology, v. 25, n. 6, p. 711-724, 1995.

CASTRO, A. A. Distribuição e longevidade de larvas infectantes de nematoides gastrintestinais de caprinos (Capra hircus) em solo e pastagem irrigados e não irrigados no município de Seropédica, RJ, Brasil. 2004. 71 f. Tese (Doutorado em Ciências Veterinárias) - Curso de Pós-graduação em Ciências Veterinárias, Universidade Federal Rural do Rio de Janeiro, 2004.

COURTNEY, C. H.; ASQUITH, R. L. Seasonal changes in pasture infectivity by equine cyathostomins in central north Florida, Equine Veterinary Journal, v. 17, n. 3, p. 240-242, 1985.

COUTO, M. C. M.; QUINELATO, S.; SANTOS, C. N.; SOUZA, L. S.; SAMPAIO, I. B. M.; RODRIGUES, M. L. A. Environmental influence in cyathostominae ecology. Veterinarni Medicina, v. 53, n. 5 , p. 243-249. 2008.

COUTO, M. C. M.; QUINELATO, S.; SOUZA, T. M.; BEVILAQUA, C. M. L.; ANJOS, D. H. S.; SAMPAIO, I. B. M.; RODRIGUES, M. L. A. Desenvolvimento e migração de larvas infectantes de ciatostomíneos (Nematoda-Cyathostominae) em gramínea cosast cross (Cynodon dactylon), em clima tropical, na Baixada Fluminense, RJ, Brasil. Revista Brasileira de Parasitologia Veterinária, v. 18, n. 2, p. 243-31-37, 2009.

CRAIG, T. M.; BOWEN, J. M.; LUDWIG, K. G. Transmission of equine cyathostomins (Strongylidae) in central Texas, American Journal of Veterinary Research, v. 44, n. 10, p. 1897-1896, 1983.

DUNCAN, J. L.; LOVE, S. Preliminary observations on an alternative strategy for the control of horse strongyles. Equine Veterinary Journal, v. 23, n. 15, p. 226-228, 1991.

EMPRESA BRASILEIRA DE PESQUISA AGROPECUÁRIA. Sistema Brasileiro de Classificação de Solos. Brasília: EMBRAPA, 2006. 412 p.

ENGLISH, A. W. Epidemiology of equine strongylosis in Southern Queesland. Part 1: the bionomics of free-living stages in feces and on pasture. Australian Veterinary Journal, v. 55, n. 7, p. 299-305, 1979a.

ENGLISH, A. W. The epidemiology of equine strongylosis in southern Queensland. Part 2: the survival and migration of infective larvae on herbage. Australian Veterinary Journal, v. 55, n. 7, p. 306-309, 1979b.

GOMEZ, H. H.; GEORGI, J. R. Equine helminth infections: control by selective chemotherapy. Equine Veterinary Journal, v. 23, n. 3, p. 198-200, 1991.

HOUSTON, R. S.; FINCHER, G. T.; CRAIG, T. M. Vertical migration of infective larvae of equine strongyles in sandy clay loam. American Journal of Veterinary Research, v. 45, n. 3, p. $575-577,1984$.

HUTCHINSON, G. W.; ABBA, S. A.; MFITILODZE, M. W. Seasonal translation of equine strongyle infective larvae to herbage in tropical Australia. Veterinary Parasitology, v. 33, n. 3-4, p. 251-263, 1989

KAPLAN, R. M. Anthelmintic resistance in nematodesof horses. Veterinary Research, v. 33, n. 5, p. 491-507, 2002.

KAPLAN, R. M. Drug resistance in nematodes of veterinary importance: a status report. Trends Parasitology, v. 20, n. 10, p. 477-481, 2004.

KRECEK, R. C.; GUTHRIE, A. J.; VAN NIEUWENHUIZEN, L. C.; BOOTH, L. M. A comparison between the effects of conventional and selective antiparasitic treatments on nematode parasites of horses from two management schemes. Journal of the South African Veterinary Association, v. 65, n. 3, p. 97-100, 1994.

KUZMINA, T. A.; KUZMIN Y. I.; KHARCHENKO, V. A. Field study on the survival, migration and overwintering of infective larvae of horse strongyles on pasture in central Ukraine, Veterinary Parasitology, v. 141, n. 3-4, p. 264-272, 2006.

LANGROVÁ, I.; JANKVSKÁ, I.; BOROVSKÝ, M.; FIALA, T. Effect of climatic influences on the migrations of infective larvae of Cyathostominae, Veterinary Medicine-Czech, v. 48, n. 1-2, p. 18-24, 2003.

LYAKU, J. R. S.; MONRAD, J.; KASSUKU, A. A. Larval ecology of bovine strongilid worms in tropical soils. Tropical Animal Health of Production, v. 20, n. 1, p. 190-192, 1998.

MATTHEE, S.; McGEOCH, M. A. Helminths in horses: use of selective treatment for the control of strongyles. Journal of the South African Veterinary Association, v. 75, n. 3, p. 129-136, 2004.

MFITILODZE, M. W.; HUTCHINSON, G. W. Development and survival of free-living stages of equine strongyles under laboratory conditions. Veterinary Parasitology, v. 23, n.1-2, p. 121-133, 1987. 
MFITILODZE, M. W.; HUTCHINSON, G. W. Development of free-living room stages of equine strongyles in faeces on pasture in the tropical environment. Veterinary Parasitolology, v. 26, n. 3-4, p. 285-296, 1988.

NIELSEN, M. K.; KAPLAN, R. M.; THAMSBORG, S. M.; MONRAAD, J.; OLSEN,S. N. Climatic influences on development and survival of free-living room stages of equine strongyles: Implications for worm control strategies and managing anthelmintic resistance. Veterinary Journal, v. 174, n. 1, p. 23-32, 2007.

OGBOURNE, C. P. Observations on the free-living stages of strongylid nematodes of horse. Parasitology, v. 64, n. 3, p. 461477, 1972.

QUINELATO, S.; COUTO, M. C. M.; RIBEIRO, B. C.; SANTOS, C. N.; SOUZA, L. S.; ANJOS, D. H. S.; SAMPAIO, I. B. M.; RODRIGUES, M. L. A. Ciatostomíneos (Strongylidae - Cyathostominae) parasitas de cavalos: ecologia experimental dos estágios pré-parasíticos em gramínea Tifton 85 (Cynodon spp. cv. tifton 85) na Baixada Fluminense, RJ, Brasil. Parasitologia Latinoamericana, v. 62, n. 1-2, p. 27-34, 2007.

QUINELATO, S.; COUTO, M. C. M.; RIBEIRO, B. C.; SANTOS, C. N.; SOUZA, L. S.; ANJOS, D. H. S.; SAMPAIO, I. B. M.; RODRIGUES, M. L. A. The ecology of horse cyathostomin infective larvae (Nematoda-Cyathostominae) in tropical southeast, Brazil. Veterinary Parasitology, v. 153, p. 100-107, 2008.

RAMSEY, Y. H.; CHISTLEY, R. M.; MATTHEWS, J. B.; HODGKINSON, J. E.; McGOLDRICK, J.; LOVE, S. Seasonal development of Cyathostominae larvae on pasture in a northern temperate region of the United Kingdom. Veterinary Parasitology, v. 119, n. 4, p. 307-318, 2004.

RODRIGUES, M. L. A.; QUINELATO, S.; COUTO,M. C. M.; SANTOS, C. N.; SOUZA, L. S.; SAMPAIO, I. B. M. Influência das condições climáticas na migração e sobrevivência de larvas infectantes de ciatostomíneos em Brachiaria humidicola, na Baixada Fluminense do Rio de Janeiro, Brasil. Ciência Animal, v. 18, n.1, p. 7-14, 2008.

ROSE, J. H.; SMALL, A. J. The distribution of infective larvae of sheep gastrointestinal nematodes in soil and on herbage and the vertical migration of Trichostrongylus vitrinus larvae through the soil. Journal of Helmintology, v. 59, n. 1, p. 127-135, 1985.

RUPASINGHE, D.; OGBOURNE, C. P. Laboratory studies on the effect of temperature on the development of the free-living stages of some strongylid nematodes of the horse. Parasitology Research, v. 55, n. 3, p. 249-253, 1978.

SAMPAIO, I. B. M. Estatística aplicada à experimentação animal. Belo Horizonte, MG: FEP MVZ Editora, 2007. 264 p.

VAN WYK, J. A. Refugia: overlooked as perhaps the most potent factor concerning the development of anthelmintic resistance. Onderstepoort Journal of Veterinary Research, v. 68, n. 1, p. 55-67, 2001.

ZAR, J. H. Biostatistical analysis. New Jersey: Prentice Hall, 1999. $663 \mathrm{p}$.

Protocolado em: 5 dez. 2008. Aceito em: 29 out. 2009. 